Supplement of Biogeosciences, 18, 1525-1541, 2021 https://doi.org/10.5194/bg-18-1525-2021-supplement (C) Author(s) 2021. This work is distributed under the Creative Commons Attribution 4.0 License.

(c) (1)

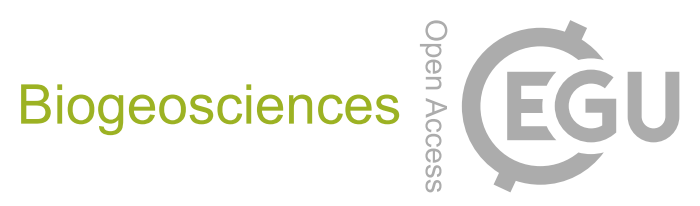

Supplement of

\title{
Factors controlling the productivity of tropical Andean forests: climate and soil are more important than tree diversity
}

\author{
Jürgen Homeier and Christoph Leuschner \\ Correspondence to: Jürgen Homeier (jhomeie@gwdg.de)
}

The copyright of individual parts of the supplement might differ from the CC BY 4.0 License. 


\begin{tabular}{|c|c|c|c|c|c|c|c|c|c|c|c|}
\hline $\begin{array}{l}\text { transe } \\
\text { ct }\end{array}$ & $\begin{array}{l}\text { plot } \\
\text { ID }\end{array}$ & site & $\begin{array}{c}\text { altitud } \\
\text { e (m) }\end{array}$ & $\begin{array}{c}\text { tree } \\
\text { species } \\
(\mathrm{dbh} \geq 10 \mathrm{c} \\
\mathrm{m})\end{array}$ & $\begin{array}{l}\text { rarefied } \\
\text { tree } \\
\text { species } \\
(\mathrm{N}=14) \\
\end{array}$ & $\begin{array}{l}\text { stems } \\
\left(\mathbf{h a}^{-1}\right)\end{array}$ & $\begin{array}{l}\text { plot } \\
\text { mean } \\
\text { WSG }\end{array}$ & $\begin{array}{l}\text { AGB } \\
(\mathbf{M g} \\
\left.\text { ha }^{-1}\right) \\
\end{array}$ & $\begin{array}{l}\text { WP } \\
(\mathrm{Mg} \\
\mathrm{ha}^{-1} \mathrm{yr}^{-} \\
\text {1) } \\
\end{array}$ & LAI & $\begin{array}{l}\mathrm{NPP} \\
(\mathrm{Mg} \\
\text { ha-1 } \\
\text { yr-1) } \\
\end{array}$ \\
\hline Loja & $\begin{array}{c}\text { BOM } \\
\text { LS1 }\end{array}$ & $\begin{array}{l}\text { Bombuscaro, } \\
\text { Podocarpus } \\
\text { NP }\end{array}$ & 1020 & 19 & 11,2 & 650 & 0,62 & 211,57 & 3,11 & 7,11 & 9,18 \\
\hline Loja & $\begin{array}{l}\text { BOM } \\
\text { LS2 }\end{array}$ & $\begin{array}{l}\text { Bombuscaro, } \\
\text { Podocarpus } \\
\text { NP }\end{array}$ & 1026 & 25 & 11,5 & 950 & 0,58 & 338,67 & 3,72 & 6,56 & 9,30 \\
\hline Loja & $\begin{array}{c}\text { BOM } \\
\text { LS3 }\end{array}$ & $\begin{array}{l}\text { Bombuscaro, } \\
\text { Podocarpus } \\
\text { NP }\end{array}$ & 1046 & 20 & 10,4 & 875 & 0,54 & 270,27 & 1,88 & NA & 6,93 \\
\hline Loja & $\begin{array}{r}\text { BOM } \\
\text { LS4 } 4\end{array}$ & $\begin{array}{l}\text { Bombuscaro, } \\
\text { Podocarpus } \\
\text { NP }\end{array}$ & 1054 & 19 & 12,0 & 625 & 0,47 & 154,71 & 4,05 & 7,48 & 9,44 \\
\hline Loja & $\begin{array}{l}\text { BOM } \\
\text { LS5 }\end{array}$ & $\begin{array}{l}\text { Bombuscaro, } \\
\text { Podocarpus } \\
\text { NP }\end{array}$ & 1056 & 13 & 9,5 & 575 & 0,52 & 218,53 & 4,36 & 7,18 & 10,24 \\
\hline Loja & $\begin{array}{l}\text { BOM } \\
\text { LS6 }\end{array}$ & $\begin{array}{l}\text { Bombuscaro, } \\
\text { Podocarpus } \\
\text { NP }\end{array}$ & 1044 & 12 & 9,6 & 625 & 0,47 & 202,27 & 2,37 & 6,88 & 7,81 \\
\hline Loja & $\begin{array}{r}\text { BOM } \\
\text { MS1 }\end{array}$ & $\begin{array}{l}\text { Bombuscaro, } \\
\text { Podocarpus } \\
\text { NP }\end{array}$ & 1132 & 19 & 11,1 & 900 & 0,61 & 156,34 & 2,96 & 5,11 & 9,08 \\
\hline Loja & $\begin{array}{r}\text { BOM } \\
\text { MS2 } \\
\end{array}$ & $\begin{array}{l}\text { Bombuscaro, } \\
\text { Podocarpus } \\
\text { NP }\end{array}$ & 1069 & 20 & 12,2 & 675 & 0,59 & 122,02 & 1,49 & 4,94 & 7,90 \\
\hline Loja & $\begin{array}{l}\text { BOM } \\
\text { MSS3 }\end{array}$ & $\begin{array}{l}\text { Bombuscaro, } \\
\text { Podocarpus } \\
\text { NP }\end{array}$ & 1049 & 18 & 9,5 & 875 & 0,52 & 171,55 & 4,72 & 5,66 & 11,46 \\
\hline Loja & $\begin{array}{c}\text { BOM } \\
\text { MSS4 }\end{array}$ & $\begin{array}{l}\text { Bombuscaro, } \\
\text { Podocarpus } \\
\text { NP }\end{array}$ & 1140 & 17 & 12,4 & 550 & 0,56 & 259,92 & 1,92 & 7,56 & 10,28 \\
\hline Loja & $\begin{array}{r}\text { BOM } \\
\_ \text {MS55 }\end{array}$ & $\begin{array}{l}\text { Bombuscaro, } \\
\text { Podocarpus } \\
\text { NP }\end{array}$ & 1170 & 20 & 12,0 & 725 & 0,54 & 179,84 & 3,00 & 6,61 & 9,36 \\
\hline Loja & $\begin{array}{c}\text { BOM } \\
\_ \text {MS6 }\end{array}$ & $\begin{array}{l}\text { Bombuscaro, } \\
\text { Podocarpus } \\
\text { NP }\end{array}$ & 1129 & 19 & 12,2 & 625 & 0,49 & 160,51 & 1,90 & 6,34 & 8,76 \\
\hline Loja & $\begin{array}{r}\text { BOM } \\
\text { US1 }\end{array}$ & $\begin{array}{l}\text { Bombuscaro, } \\
\text { Podocarpus } \\
\text { NP }\end{array}$ & 1075 & 10 & 5,1 & 1100 & 0,62 & 154,04 & 2,16 & 5,82 & 5,79 \\
\hline Loja & $\begin{array}{r}\text { BOM } \\
\text { US2 }\end{array}$ & $\begin{array}{l}\text { Bombuscaro, } \\
\text { Podocarpus } \\
\text { NP }\end{array}$ & 1066 & 10 & 5,4 & 875 & 0,60 & 84,27 & 1,05 & 5,53 & 5,53 \\
\hline Loja & $\begin{array}{r}\text { BOM } \\
\text { US3 }\end{array}$ & $\begin{array}{l}\text { Bombuscaro, } \\
\text { Podocarpus } \\
\text { NP }\end{array}$ & 1072 & 10 & 7,6 & 725 & 0,59 & 89,37 & 1,07 & 7,15 & 5,49 \\
\hline Loja & $\begin{array}{r}\text { BOM } \\
\text { US4 }\end{array}$ & $\begin{array}{l}\text { Bombuscaro, } \\
\text { Podocarpus } \\
\text { NP }\end{array}$ & 1268 & 16 & 10,5 & 650 & 0,53 & 143,11 & 2,09 & 3,26 & 8,17 \\
\hline Loja & $\begin{array}{l}\text { BOM } \\
\text { US5 }\end{array}$ & $\begin{array}{l}\text { Bombuscaro, } \\
\text { Podocarpus } \\
\text { NP }\end{array}$ & 1257 & 21 & 11,3 & 825 & 0,52 & 226,05 & 4,95 & 5,89 & 10,62 \\
\hline Loja & $\begin{array}{l}\text { BOM } \\
\text { US6 }\end{array}$ & $\begin{array}{l}\text { Bombuscaro, } \\
\text { Podocarpus } \\
\text { NP }\end{array}$ & 1266 & 18 & 11,1 & 700 & 0,54 & 142,34 & 4,23 & 6,86 & 9,31 \\
\hline Loja & $\begin{array}{l}\mathrm{CAJ}_{-} \\
\text {LS1 }\end{array}$ & $\begin{array}{l}\text { Cajanuma, } \\
\text { Podocarpus } \\
\text { NP }\end{array}$ & 2818 & 19 & 13,5 & 500 & 0,55 & 166,36 & 0,87 & 5,51 & 5,20 \\
\hline
\end{tabular}




\begin{tabular}{|c|c|c|c|c|c|c|c|c|c|c|c|}
\hline Loja & $\begin{array}{l}\mathrm{CAJ}_{-} \\
\mathrm{LS} 2\end{array}$ & $\begin{array}{l}\text { Cajanuma, } \\
\text { Podocarpus } \\
\text { NP }\end{array}$ & 2805 & 11 & 9,7 & 400 & 0,57 & 96,76 & 0,82 & 5,64 & 4,53 \\
\hline Loja & $\begin{array}{l}\mathrm{CAJ}_{-} \\
\mathrm{LS} 3\end{array}$ & $\begin{array}{l}\text { Cajanuma, } \\
\text { Podocarpus } \\
\text { NP }\end{array}$ & 2798 & 12 & 8,7 & 500 & 0,56 & 182,70 & 1,22 & 3,77 & 4,88 \\
\hline Loja & $\begin{array}{l}\text { CAJ_- } \\
\text { LS4 }\end{array}$ & $\begin{array}{l}\text { Cajanuma, } \\
\text { Podocarpus } \\
\text { NP }\end{array}$ & 2865 & 19 & 9,8 & 1100 & 0,59 & 157,91 & 0,50 & 6,85 & 3,89 \\
\hline Loja & $\begin{array}{l}\mathrm{CAJ}_{-} \\
\mathrm{LS} 5\end{array}$ & $\begin{array}{l}\text { Cajanuma, } \\
\text { Podocarpus } \\
\text { NP }\end{array}$ & 2878 & 21 & 9,2 & 1275 & 0,54 & 98,82 & 1,37 & 5,8 & 5,04 \\
\hline Loja & $\begin{array}{l}\mathrm{CAJ}_{-} \\
\mathrm{LS6}\end{array}$ & $\begin{array}{l}\text { Cajanuma, } \\
\text { Podocarpus } \\
\text { NP }\end{array}$ & 2880 & 27 & 11,1 & 1075 & 0,53 & 89,36 & 0,49 & 7,13 & 4,11 \\
\hline Loja & $\begin{array}{l}\mathrm{CAJ}_{-} \\
\mathrm{MS} 1\end{array}$ & $\begin{array}{l}\text { Cajanuma, } \\
\text { Podocarpus } \\
\text { NP }\end{array}$ & 2859 & 20 & 9,5 & 1375 & 0,59 & 79,77 & 0,52 & 4,61 & 4,01 \\
\hline Loja & $\begin{array}{l}\mathrm{CAJ}_{-} \\
\mathrm{MS} 2\end{array}$ & $\begin{array}{l}\text { Cajanuma, } \\
\text { Podocarpus } \\
\text { NP }\end{array}$ & 2872 & 17 & 8,4 & 1175 & 0,64 & 80,74 & 0,48 & 4,4 & 5,59 \\
\hline Loja & $\begin{array}{l}\mathrm{CAJ}_{-} \\
\mathrm{MS} 3\end{array}$ & $\begin{array}{l}\text { Cajanuma, } \\
\text { Podocarpus } \\
\text { NP }\end{array}$ & 2844 & 20 & 9,4 & 1450 & 0,57 & 103,76 & 1,38 & 4,68 & 5,89 \\
\hline Loja & $\begin{array}{l}\mathrm{CAJ}_{-} \\
\mathrm{MS} 4\end{array}$ & $\begin{array}{l}\text { Cajanuma, } \\
\text { Podocarpus } \\
\text { NP }\end{array}$ & 2900 & 14 & 10,6 & 525 & 0,61 & 45,04 & 0,22 & 5,34 & 3,62 \\
\hline Loja & $\begin{array}{l}\mathrm{CAJ}_{-} \\
\mathrm{MS} 5\end{array}$ & $\begin{array}{l}\text { Cajanuma, } \\
\text { Podocarpus } \\
\text { NP }\end{array}$ & 2885 & 21 & 10,8 & 725 & 0,59 & 97,10 & 0,56 & 4,84 & 4,78 \\
\hline Loja & $\begin{array}{l}\mathrm{CAJ}_{-} \\
\mathrm{MS6}\end{array}$ & $\begin{array}{l}\text { Cajanuma, } \\
\text { Podocarpus } \\
\text { NP }\end{array}$ & 2875 & 13 & 8,9 & 950 & 0,61 & 49,66 & 0,38 & 3,93 & 3,15 \\
\hline Loja & $\begin{array}{l}\text { CAJ_- } \\
\text { US1 }\end{array}$ & $\begin{array}{l}\text { Cajanuma, } \\
\text { Podocarpus } \\
\text { NP }\end{array}$ & 2891 & 25 & 10,7 & 1025 & 0,59 & 74,67 & 0,36 & 3,07 & 3,51 \\
\hline Loja & $\begin{array}{l}\mathrm{CAJ}_{-} \\
\mathrm{US} 2\end{array}$ & $\begin{array}{l}\text { Cajanuma, } \\
\text { Podocarpus } \\
\text { NP }\end{array}$ & 2885 & 18 & 9,5 & 1025 & 0,64 & 49,03 & 0,41 & 3,58 & 3,46 \\
\hline Loja & $\begin{array}{l}\text { CAJ_- } \\
\text { US3 }\end{array}$ & $\begin{array}{l}\text { Cajanuma, } \\
\text { Podocarpus } \\
\text { NP }\end{array}$ & 2869 & 13 & 7,6 & 1475 & 0,59 & 71,30 & 0,43 & 4,69 & 4,33 \\
\hline Loja & $\begin{array}{l}\mathrm{CAJ}_{-} \\
\mathrm{US} 4\end{array}$ & $\begin{array}{l}\text { Cajanuma, } \\
\text { Podocarpus } \\
\text { NP }\end{array}$ & 2886 & 17 & 9,3 & 1075 & 0,60 & 51,49 & 0,83 & 6,18 & 3,95 \\
\hline Loja & $\begin{array}{l}\text { CAJ_- } \\
\text { US5 }\end{array}$ & $\begin{array}{l}\text { Cajanuma, } \\
\text { Podocarpus } \\
\text { NP }\end{array}$ & 2890 & 18 & 8,6 & 1325 & 0,58 & 68,69 & 0,46 & 4,88 & 4,05 \\
\hline Loja & $\begin{array}{l}\text { CAJ_- } \\
\text { US6 }\end{array}$ & $\begin{array}{l}\text { Cajanuma, } \\
\text { Podocarpus } \\
\text { NP }\end{array}$ & 2893 & 15 & 8,2 & 1025 & 0,61 & 45,39 & 0,45 & 5,49 & 3,96 \\
\hline Loja & $\begin{array}{l}\text { SFM_ } \\
\text { LS1 }\end{array}$ & $\begin{array}{l}\text { San Francisco } \\
\text { Reserve }\end{array}$ & 2039 & 20 & 12,1 & 650 & 0,49 & 274,45 & 6.51 & 4,71 & 13,39 \\
\hline Loja & $\begin{array}{l}\text { SFM_ } \\
\text { LS2 }\end{array}$ & $\begin{array}{l}\text { San Francisco } \\
\text { Reserve }\end{array}$ & 1993 & 12 & 10,6 & 400 & 0,47 & 272,54 & 3,35 & 6,09 & 10,32 \\
\hline Loja & $\begin{array}{l}\text { SFM_ } \\
\text { LS3 }\end{array}$ & $\begin{array}{l}\text { San Francisco } \\
\text { Reserve }\end{array}$ & 2020 & 14 & 10,2 & 550 & 0,47 & 191,14 & 2,41 & 5,61 & 8,39 \\
\hline Loja & $\begin{array}{l}\text { SFM_ } \\
\text { LS4 }\end{array}$ & $\begin{array}{l}\text { San Francisco } \\
\text { Reserve }\end{array}$ & 1913 & 12 & 9,6 & 925 & 0,49 & 29,83 & 0,80 & 9,35 & 7,56 \\
\hline Loja & $\begin{array}{l}\text { SFM } \\
\text { LS5 }\end{array}$ & $\begin{array}{l}\text { San Francisco } \\
\text { Reserve }\end{array}$ & 1954 & 25 & 11,9 & 825 & 0,49 & 147,86 & 3,34 & 8,6 & 12,06 \\
\hline Loja & $\begin{array}{l}\text { SFM_ } \\
\text { LS6 }\end{array}$ & $\begin{array}{l}\text { San Francisco } \\
\text { Reserve }\end{array}$ & 1933 & 25 & 12,3 & 475 & 0,52 & 423,87 & 2,46 & 7,04 & 10,92 \\
\hline Loja & $\begin{array}{l}\text { SFM_ } \\
\text { MS1 }\end{array}$ & $\begin{array}{l}\text { San Francisco } \\
\text { Reserve }\end{array}$ & 2034 & 21 & 12,1 & 775 & 0,55 & 119,30 & 1,46 & 5,93 & 7,14 \\
\hline
\end{tabular}




\begin{tabular}{|c|c|c|c|c|c|c|c|c|c|c|c|}
\hline Loja & $\begin{array}{l}\text { SFM_- } \\
\text { MS2 }\end{array}$ & $\begin{array}{l}\text { San Francisco } \\
\text { Reserve }\end{array}$ & 1950 & 27 & 12,4 & 825 & 0,52 & 132,93 & 2,03 & 8,03 & 10,77 \\
\hline Loja & $\begin{array}{l}\text { SFM_ } \\
\text { MS3 }\end{array}$ & $\begin{array}{l}\text { San Francisco } \\
\text { Reserve }\end{array}$ & 2027 & 18 & 11,5 & 775 & 0,58 & 229,94 & 1,71 & 2,83 & 9,50 \\
\hline Loja & $\begin{array}{l}\text { SFM_ } \\
\text { MS4 }\end{array}$ & $\begin{array}{l}\text { San Francisco } \\
\text { Reserve }\end{array}$ & 1927 & 26 & 11,5 & 1100 & 0,47 & 132,24 & 2,80 & 7,18 & 10,94 \\
\hline Loja & $\begin{array}{l}\text { SFM_ } \\
\text { MS5 }\end{array}$ & $\begin{array}{l}\text { San Francisco } \\
\text { Reserve }\end{array}$ & 1971 & 20 & 10,0 & 925 & 0,48 & 104,30 & 2,63 & 9,68 & 11,82 \\
\hline Loja & $\begin{array}{l}\text { SFM_ } \\
\text { MS6 }\end{array}$ & $\begin{array}{l}\text { San Francisco } \\
\text { Reserve }\end{array}$ & 2020 & 24 & 12,2 & 700 & 0,47 & 94,62 & 1,53 & 8,67 & 8,30 \\
\hline Loja & $\begin{array}{l}\text { SFM_ } \\
\text { US1 }\end{array}$ & $\begin{array}{l}\text { San Francisco } \\
\text { Reserve }\end{array}$ & 2002 & 14 & 9,6 & 775 & 0,57 & 61,50 & 1,13 & 5,94 & 6,62 \\
\hline Loja & $\begin{array}{l}\text { SFM_ } \\
\text { US2 }\end{array}$ & $\begin{array}{l}\text { San Francisco } \\
\text { Reserve }\end{array}$ & 2026 & 22 & 11,0 & 1250 & 0,55 & 142,20 & 2,15 & 5,54 & 8,00 \\
\hline Loja & $\begin{array}{l}\text { SFM_ } \\
\text { US3 }\end{array}$ & $\begin{array}{l}\text { San Francisco } \\
\text { Reserve }\end{array}$ & 2089 & 18 & 9,0 & 1000 & 0,52 & 83,38 & 1,24 & 5,03 & 6,83 \\
\hline Loja & $\begin{array}{l}\text { SFM_ } \\
\text { US4 }\end{array}$ & $\begin{array}{l}\text { San Francisco } \\
\text { Reserve }\end{array}$ & 2063 & 23 & 10,7 & 1200 & 0,57 & 139,32 & 0,90 & 8,37 & 5,39 \\
\hline Loja & $\begin{array}{l}\text { SFM_ } \\
\text { US5 }\end{array}$ & $\begin{array}{l}\text { San Francisco } \\
\text { Reserve }\end{array}$ & 2054 & 24 & 11,9 & 850 & 0,54 & 112,28 & 1,07 & 6,13 & 6,31 \\
\hline Loja & $\begin{array}{l}\text { SFM_ } \\
\text { US6 }\end{array}$ & $\begin{array}{l}\text { San Francisco } \\
\text { Reserve }\end{array}$ & 2039 & 24 & 10,9 & 1025 & 0,56 & 152,56 & 1,19 & 4,63 & 6,53 \\
\hline Napo & $\begin{array}{c}\mathrm{YAY}_{-} \\
01\end{array}$ & $\begin{array}{l}\text { Yanayacu } \\
\text { biological } \\
\text { station, } \\
\text { Reserva } \\
\text { Biósfera } \\
\text { Sumaco }\end{array}$ & 2085 & 12 & 9,1 & 575 & 0,40 & 189,73 & 1,83 & 5,418 & NA \\
\hline Napo & $\begin{array}{c}\mathrm{YAY}_{-} \\
02\end{array}$ & $\begin{array}{l}\text { Yanayacu } \\
\text { biological } \\
\text { station, } \\
\text { Reserva } \\
\text { Biósfera } \\
\text { Sumaco }\end{array}$ & 2080 & 14 & 9,1 & 725 & 0,47 & 312,37 & $\mathrm{NA}$ & 5,223 & NA \\
\hline Napo & $\begin{array}{c}\mathrm{YAY}_{-} \\
03\end{array}$ & $\begin{array}{l}\text { Yanayacu } \\
\text { biological } \\
\text { station, } \\
\text { Reserva } \\
\text { Biósfera } \\
\text { Sumaco } \\
\end{array}$ & 2055 & 16 & 9,1 & 800 & 0,52 & 407,38 & 6,09 & 4,592 & NA \\
\hline Napo & $\begin{array}{c}\mathrm{YAY}_{-} \\
04\end{array}$ & $\begin{array}{l}\text { Yanayacu } \\
\text { biological } \\
\text { station, } \\
\text { Reserva } \\
\text { Biósfera } \\
\text { Sumaco }\end{array}$ & 2070 & 13 & 9,5 & 625 & 0,51 & 259,40 & NA & 5,065 & NA \\
\hline Napo & $\begin{array}{c}\mathrm{YAY}_{-} \\
05\end{array}$ & $\begin{array}{l}\text { Yanayacu } \\
\text { biological } \\
\text { station, } \\
\text { Reserva } \\
\text { Biósfera } \\
\text { Sumaco }\end{array}$ & 2080 & 19 & 10,2 & 800 & 0,44 & 440,00 & NA & 4,327 & NA \\
\hline Napo & $\begin{array}{c}\text { GUA_ } \\
06\end{array}$ & $\begin{array}{l}\text { Cordillera } \\
\text { Guacamayos, } \\
\text { Reserva } \\
\text { Ecológica } \\
\text { Antisana }\end{array}$ & 2000 & 18 & 11,2 & 700 & 0,55 & 113,87 & 2,60 & 5,803 & NA \\
\hline Napo & $\begin{array}{c}\mathrm{GUA}_{-} \\
07\end{array}$ & $\begin{array}{l}\text { Cordillera } \\
\text { Guacamayos, } \\
\text { Reserva } \\
\text { Ecológica } \\
\text { Antisana }\end{array}$ & 1940 & 19 & 11,0 & 675 & 0,61 & 168,32 & 2,48 & 4 & NA \\
\hline Napo & $\begin{array}{c}\text { GUA_ } \\
08\end{array}$ & $\begin{array}{l}\text { Cordillera } \\
\text { Guacamayos, } \\
\text { Reserva }\end{array}$ & 1995 & 14 & 10,2 & 575 & 0,48 & 121,53 & 1,87 & 5,267 & NA \\
\hline
\end{tabular}




\begin{tabular}{|c|c|c|c|c|c|c|c|c|c|c|c|}
\hline & & $\begin{array}{l}\text { Ecológica } \\
\text { Antisana }\end{array}$ & & & & & & & & & \\
\hline Napo & $\begin{array}{c}\text { SUM_ }_{09} \\
09\end{array}$ & $\begin{array}{l}\text { Sumaco } \\
\text { volcano, PN } \\
\text { Sumaco- } \\
\text { Galeras }\end{array}$ & 1920 & 6 & 6,0 & 400 & 0,53 & 239,26 & 2,19 & 5,493 & NA \\
\hline Napo & $\begin{array}{c}\mathrm{SUM}_{-} \\
10\end{array}$ & $\begin{array}{l}\text { Sumaco } \\
\text { volcano, PN } \\
\text { Sumaco- } \\
\text { Galeras }\end{array}$ & 1940 & 13 & 9,8 & 525 & 0,53 & 458,75 & NA & 5,805 & NA \\
\hline Napo & $\begin{array}{l}\mathrm{SUM}_{1} \\
11\end{array}$ & $\begin{array}{l}\text { Sumaco } \\
\text { volcano, PN } \\
\text { Sumaco- } \\
\text { Galeras }\end{array}$ & 1950 & 13 & 10,5 & 475 & 0,53 & 129,13 & 3,79 & 4,848 & NA \\
\hline Napo & $\begin{array}{l}\mathrm{SUM}_{1} \\
12\end{array}$ & $\begin{array}{l}\text { Sumaco } \\
\text { volcano, PN } \\
\text { Sumaco- } \\
\text { Galeras }\end{array}$ & 2015 & 20 & 11,0 & 800 & 0,50 & 907,54 & 4,38 & 4,226 & NA \\
\hline Napo & $\begin{array}{c}\mathrm{SUM}_{13} \\
-\end{array}$ & $\begin{array}{l}\text { Sumaco } \\
\text { volcano, PN } \\
\text { Sumaco- } \\
\text { Galeras }\end{array}$ & 2000 & 18 & 11,7 & 625 & 0,52 & 535,74 & NA & 5,179 & NA \\
\hline Napo & $\begin{array}{c}\mathrm{SUM}_{14} \\
14\end{array}$ & $\begin{array}{l}\text { Sumaco } \\
\text { volcano, PN } \\
\text { Sumaco- } \\
\text { Galeras }\end{array}$ & 1580 & 16 & 11,2 & 475 & 0,54 & 287,58 & NA & 4,935 & NA \\
\hline Napo & $\begin{array}{c}\mathrm{SUM}_{15} \\
15\end{array}$ & $\begin{array}{l}\text { Sumaco } \\
\text { volcano, PN } \\
\text { Sumaco- } \\
\text { Galeras }\end{array}$ & 1590 & 16 & 11,5 & 550 & 0,59 & 393,05 & 3,82 & 4,486 & NA \\
\hline Napo & $\begin{array}{l}\mathrm{SUM}_{-} \\
16\end{array}$ & $\begin{array}{l}\text { Sumaco } \\
\text { volcano, PN } \\
\text { Sumaco- } \\
\text { Galeras }\end{array}$ & 1610 & 16 & 10,5 & 625 & 0,61 & 286,80 & 4,15 & 5,294 & NA \\
\hline Napo & $\begin{array}{c}\mathrm{SUM}_{17} \\
-\end{array}$ & $\begin{array}{l}\text { Sumaco } \\
\text { volcano, PN } \\
\text { Sumaco- } \\
\text { Galeras }\end{array}$ & 1610 & 15 & 10,2 & 625 & 0,55 & 528,21 & NA & 4,212 & NA \\
\hline Napo & $\begin{array}{c}\mathrm{SUM}_{1} \\
18\end{array}$ & $\begin{array}{l}\text { Sumaco } \\
\text { volcano, PN } \\
\text { Sumaco- } \\
\text { Galeras }\end{array}$ & 1590 & 15 & 10.3 & 525 & 0,57 & 670,66 & 138 & 531 & NA \\
\hline Napo & $\begin{array}{l}\mathrm{SUM}_{-} \\
19\end{array}$ & $\begin{array}{l}\text { Sumaco } \\
\text { volcano, PN } \\
\text { Sumaco- } \\
\text { Galeras }\end{array}$ & 1590 & 20 & 12,5 & 600 & 0,54 & 439,45 & NA & 5,01 & NA \\
\hline Napo & $\begin{array}{c}\mathrm{GAL}_{-} \\
20\end{array}$ & $\begin{array}{l}\text { Cordillera } \\
\text { Galeras, PN } \\
\text { Sumaco- } \\
\text { Galeras }\end{array}$ & 1090 & 19 & 11,1 & 725 & 0,55 & 488,16 & 4,08 & 4,611 & NA \\
\hline Napo & $\begin{array}{c}\mathrm{GAL}_{-} \\
21\end{array}$ & $\begin{array}{l}\text { Cordillera } \\
\text { Galeras, PN } \\
\text { Sumaco- } \\
\text { Galeras }\end{array}$ & 1080 & 11 & 8,8 & 475 & 0,47 & 286.16 & NA & 4.223 & NA \\
\hline Napo & $\mathrm{GAL}_{22}$ & $\begin{array}{l}\text { Cordillera } \\
\text { Galeras, PN } \\
\text { Sumaco- } \\
\text { Galeras }\end{array}$ & 1060 & 24 & 11,5 & 825 & 0,51 & 217,59 & 3,55 & 4,331 & NA \\
\hline Napo & $\begin{array}{c}\mathrm{GAL}_{-} \\
23\end{array}$ & $\begin{array}{l}\text { Cordillera } \\
\text { Galeras, PN } \\
\text { Sumaco- } \\
\text { Galeras }\end{array}$ & 1090 & 23 & 13,0 & 675 & 0,62 & 586,32 & NA & 4,846 & NA \\
\hline Napo & $\begin{array}{c}\mathrm{GAL}_{-} \\
24\end{array}$ & $\begin{array}{l}\text { Cordillera } \\
\text { Galeras, PN } \\
\text { Sumaco- } \\
\text { Galeras }\end{array}$ & 1110 & 24 & 12,0 & 825 & 0,57 & 231,01 & 4,13 & 4,35 & NA \\
\hline
\end{tabular}




\begin{tabular}{|c|c|c|c|c|c|c|c|c|c|c|c|}
\hline Napo & $\begin{array}{c}\mathrm{GAL}_{-} \\
25 \\
\end{array}$ & $\begin{array}{l}\text { Cordillera } \\
\text { Galeras, PN } \\
\text { Sumaco- } \\
\text { Galeras } \\
\end{array}$ & 1450 & 27 & 11,8 & 950 & 0,60 & 343,35 & 2,43 & 5,049 & NA \\
\hline Napo & $\begin{array}{c}\mathrm{GAL}_{-} \\
26\end{array}$ & $\begin{array}{l}\text { Cordillera } \\
\text { Galeras, PN } \\
\text { Sumaco- } \\
\text { Galeras } \\
\end{array}$ & 1560 & 24 & 11,5 & 800 & 0,58 & 305,02 & 2,19 & 6,053 & NA \\
\hline Napo & $\begin{array}{c}\text { GUA_- } \\
27\end{array}$ & $\begin{array}{l}\text { Cordillera } \\
\text { Guacamayos, } \\
\text { Reserva } \\
\text { Ecológica } \\
\text { Antisana }\end{array}$ & 1990 & 22 & 11,7 & 775 & 0,65 & 227,73 & NA & 5,392 & NA \\
\hline Napo & $\begin{array}{c}\mathrm{GAL}_{-} \\
28\end{array}$ & $\begin{array}{l}\text { Cordillera } \\
\text { Galeras, PN } \\
\text { Sumaco- } \\
\text { Galeras } \\
\end{array}$ & 1597 & 26 & 10,8 & 1125 & 0,59 & 235,28 & NA & 6,185 & NA \\
\hline Napo & $\begin{array}{c}\mathrm{GAL}_{-} \\
29\end{array}$ & $\begin{array}{l}\text { Cordillera } \\
\text { Galeras, PN } \\
\text { Sumaco- } \\
\text { Galeras }\end{array}$ & 1050 & 25 & 11,8 & 900 & 0,57 & 310,39 & NA & 5,114 & NA \\
\hline Napo & $\begin{array}{c}\text { HAM } \\
-42 \\
\end{array}$ & $\begin{array}{l}\text { Hakuna } \\
\text { Matata, } \\
\text { Reserva } \\
\text { Biósfera } \\
\text { Sumaco }\end{array}$ & 970 & 17 & 10,2 & 1050 & 0,63 & 555,75 & 5,68 & 7,681 & NA \\
\hline Napo & $\begin{array}{c}\text { HAM } \\
-43 \\
\end{array}$ & $\begin{array}{l}\text { Hakuna } \\
\text { Matata, } \\
\text { Reserva } \\
\text { Biósfera } \\
\text { Sumaco } \\
\end{array}$ & 960 & 27 & 13,2 & 950 & 0,61 & 391,57 & 3,83 & 7,444 & NA \\
\hline Napo & $\begin{array}{c}\text { HAM } \\
-44 \\
\end{array}$ & $\begin{array}{l}\text { Hakuna } \\
\text { Matata, } \\
\text { Reserva } \\
\text { Biósfera } \\
\text { Sumaco }\end{array}$ & 1000 & 21 & 11,4 & 850 & 0,61 & 391,63 & NA & 6,813 & NA \\
\hline Napo & $\begin{array}{c}\text { HAM } \\
-45\end{array}$ & $\begin{array}{l}\text { Hakuna } \\
\text { Matata, } \\
\text { Reserva } \\
\text { Biósfera } \\
\text { Sumaco }\end{array}$ & 1050 & 27 & 12,1 & 900 & 0,60 & 339,33 & NA & 7,239 & NA \\
\hline Napo & $\begin{array}{c}\text { HAM } \\
-46 \\
\end{array}$ & $\begin{array}{l}\text { Hakuna } \\
\text { Matata, } \\
\text { Reserva } \\
\text { Biósfera } \\
\text { Sumaco }\end{array}$ & 1020 & 27 & 13,1 & 1000 & 0,63 & 451,42 & NA & 7,854 & NA \\
\hline Napo & $\begin{array}{c}\mathrm{GAL}_{-} \\
47\end{array}$ & $\begin{array}{l}\text { Cordillera } \\
\text { Galeras, PN } \\
\text { Sumaco- } \\
\text { Galeras } \\
\end{array}$ & 1000 & 18 & 12,4 & 600 & 0,52 & 183,17 & NA & 5,514 & NA \\
\hline Napo & $\begin{array}{c}\mathrm{GAL}_{-} \\
48\end{array}$ & $\begin{array}{l}\text { Cordillera } \\
\text { Galeras, PN } \\
\text { Sumaco- } \\
\text { Galeras } \\
\end{array}$ & 1130 & 23 & 12,7 & 725 & 0,51 & 224,37 & NA & 4,874 & NA \\
\hline Napo & $\begin{array}{c}\mathrm{GAL}_{-} \\
49\end{array}$ & $\begin{array}{l}\text { Cordillera } \\
\text { Galeras, PN } \\
\text { Sumaco- } \\
\text { Galeras }\end{array}$ & 1080 & 23 & 11,4 & 850 & 0,54 & 304,24 & NA & 4,939 & NA \\
\hline Napo & $\begin{array}{c}\mathrm{GAL}_{-} \\
50\end{array}$ & $\begin{array}{l}\text { Cordillera } \\
\text { Galeras, PN } \\
\text { Sumaco- } \\
\text { Galeras }\end{array}$ & 1557 & 30 & 12,3 & 1200 & 0,63 & 296,53 & NA & 6,034 & NA \\
\hline Napo & $\begin{array}{c}\text { HAM } \\
51 \\
\end{array}$ & $\begin{array}{l}\text { Hakuna } \\
\text { Matata, } \\
\text { Reserva }\end{array}$ & 1080 & 28 & 13,5 & 850 & 0,62 & 560,12 & NA & 7,966 & NA \\
\hline
\end{tabular}




\begin{tabular}{|c|c|c|c|c|c|c|c|c|c|c|c|}
\hline & & $\begin{array}{l}\text { Biósfera } \\
\text { Sumaco }\end{array}$ & & & & & & & & & \\
\hline Napo & $\begin{array}{c}\mathrm{GUA}_{-} \\
52\end{array}$ & $\begin{array}{l}\text { Cordillera } \\
\text { Guacamayos, } \\
\text { Reserva } \\
\text { Ecológica } \\
\text { Antisana }\end{array}$ & 1980 & 14 & 8,9 & 925 & 0,57 & 168,89 & NA & 4,267 & NA \\
\hline Napo & $\begin{array}{c}\mathrm{GUA}_{5} \\
-\end{array}$ & $\begin{array}{l}\text { Cordillera } \\
\text { Guacamayos, } \\
\text { Reserva } \\
\text { Ecológica } \\
\text { Antisana }\end{array}$ & 1980 & 26 & 12,1 & 1025 & 0,64 & 221,89 & NA & 5,568 & NA \\
\hline Napo & $\begin{array}{c}\mathrm{GUA}_{5} \\
-\end{array}$ & $\begin{array}{l}\text { Cordillera } \\
\text { Guacamayos, } \\
\text { Reserva } \\
\text { Ecológica } \\
\text { Antisana }\end{array}$ & 2000 & 25 & 12,7 & 800 & 0,59 & 317,10 & NA & 6,324 & NA \\
\hline Napo & $\begin{array}{c}\mathrm{GUA}_{-} \\
55\end{array}$ & $\begin{array}{l}\text { Cordillera } \\
\text { Guacamayos, } \\
\text { Reserva } \\
\text { Ecológica } \\
\text { Antisana } \\
\end{array}$ & 2000 & 21 & 11,5 & 825 & 0,58 & 294,81 & NA & 6,021 & NA \\
\hline Napo & $\begin{array}{c}\text { SUM }_{-} \\
56\end{array}$ & $\begin{array}{l}\text { Sumaco } \\
\text { volcano, PN } \\
\text { Sumaco- } \\
\text { Galeras } \\
\end{array}$ & 1931 & 25 & 12,3 & 875 & 0,59 & 356,77 & NA & 4,904 & NA \\
\hline Napo & $\begin{array}{c}\mathrm{SUM}_{57} \\
\end{array}$ & $\begin{array}{l}\text { Sumaco } \\
\text { volcano, PN } \\
\text { Sumaco-- } \\
\text { Galeras } \\
\end{array}$ & 2000 & 11 & 6,6 & 725 & 0,51 & 268,77 & NA & 4,811 & NA \\
\hline Napo & $\begin{array}{c}\mathrm{SUM}_{-} \\
58\end{array}$ & $\begin{array}{l}\text { Sumaco } \\
\text { volcano, PN } \\
\text { Sumaco- } \\
\text { Galeras } \\
\end{array}$ & 1580 & 15 & 11,2 & 675 & 0,56 & 272,36 & NA & 4,339 & NA \\
\hline Napo & $\begin{array}{c}\mathrm{SUM}_{5} \\
-\end{array}$ & $\begin{array}{l}\text { Sumaco } \\
\text { volcano, PN } \\
\text { Sumaco- } \\
\text { Galeras }\end{array}$ & 1600 & 21 & 12,3 & 725 & 0,55 & 537,08 & NA & 5,915 & NA \\
\hline Napo & $\begin{array}{c}\mathrm{GAL}_{-} \\
60\end{array}$ & $\begin{array}{l}\text { Cordillera } \\
\text { Galeras, PN } \\
\text { Sumaco- } \\
\text { Galeras }\end{array}$ & 1570 & 26 & 12,6 & 925 & 0,68 & 255,79 & 2,33 & 6,006 & NA \\
\hline Napo & $\begin{array}{c}\mathrm{GAL}_{-} \\
61\end{array}$ & $\begin{array}{l}\text { Cordillera } \\
\text { Galeras, PN } \\
\text { Sumaco- } \\
\text { Galeras }\end{array}$ & 1560 & 22 & 12,2 & 825 & 0,67 & 451,96 & NA & 6,77 & NA \\
\hline Napo & $\begin{array}{c}\mathrm{GAL}_{-} \\
62\end{array}$ & $\begin{array}{l}\text { Cordillera } \\
\text { Galeras, PN } \\
\text { Sumaco- } \\
\text { Galeras }\end{array}$ & 1590 & 23 & 10,7 & 1200 & 0,60 & 254,85 & NA & 5,712 & NA \\
\hline Napo & ${ }_{63}^{\mathrm{RHO}_{-}}$ & $\begin{array}{l}\text { Rio Hollín, } \\
\text { Reserva } \\
\text { Biósfera } \\
\text { Sumaco }\end{array}$ & 1190 & 15 & 7,2 & 1100 & 0,48 & 337,46 & NA & 5,93 & NA \\
\hline Napo & $\mathrm{RHO}_{64}$ & $\begin{array}{l}\text { Rio Hollín, } \\
\text { Reserva } \\
\text { Biósfera } \\
\text { Sumaco }\end{array}$ & 1195 & 13 & 5,8 & 1150 & 0,44 & 146,24 & 6,67 & 6,551 & NA \\
\hline Napo & $\mathrm{RHO}_{65}$ & $\begin{array}{l}\text { Rio Hollín, } \\
\text { Reserva } \\
\text { Biósfera } \\
\text { Sumaco }\end{array}$ & 1210 & 24 & 11,0 & 950 & 0,55 & 893,95 & 5,93 & 7,51 & NA \\
\hline Napo & $\mathrm{RHO}_{66}$ & $\begin{array}{l}\text { Rio Hollín, } \\
\text { Reserva } \\
\text { Biósfera } \\
\text { Sumaco }\end{array}$ & 1165 & 19 & 8,2 & 1150 & 0,45 & 202,43 & 6,88 & 6,387 & NA \\
\hline
\end{tabular}




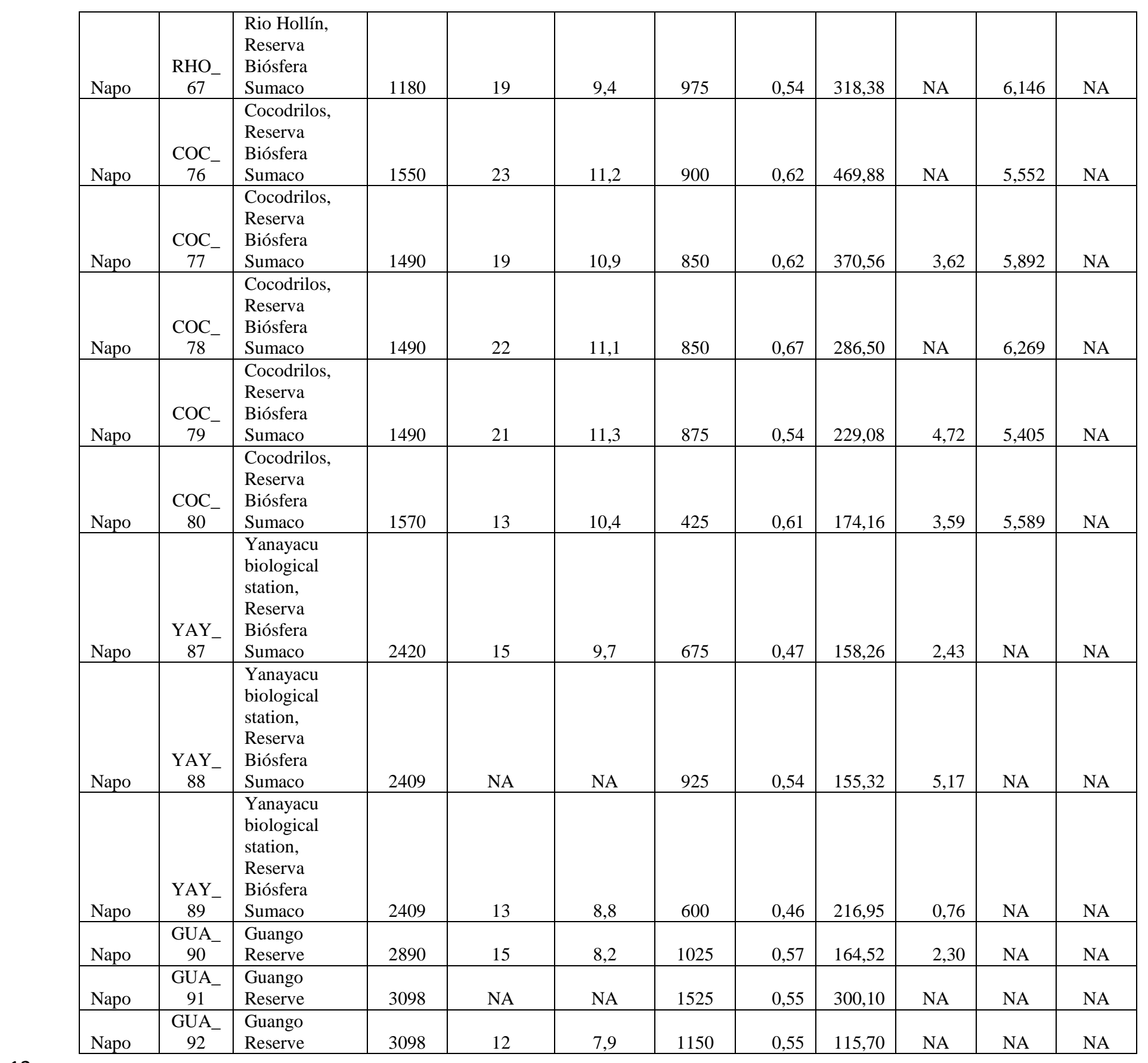


Supplement S2: Plot soil parameters

16

\begin{tabular}{|c|c|c|c|c|c|c|c|c|c|c|c|c|c|}
\hline $\begin{array}{l}\text { transec } \\
\mathrm{t}\end{array}$ & plot ID & site & $\begin{array}{c}\text { altitude } \\
(\mathbf{m})\end{array}$ & $\begin{array}{l}\text { organic } \\
\text { layer } \\
\text { depth } \\
(\mathrm{cm}) \\
\end{array}$ & $\begin{array}{l}\text { pH } \\
(\mathrm{KCl})\end{array}$ & $\begin{array}{l}\mathbf{K} \\
(\mu \mathrm{mol} / \mathrm{g})\end{array}$ & $\begin{array}{l}\mathrm{Mg} \\
(\mu \mathrm{mol} / \mathrm{g})\end{array}$ & $\begin{array}{l}\mathrm{Ca} \\
(\mu \mathrm{mol} / \mathrm{g})\end{array}$ & $\begin{array}{l}\text { Al } \\
(\mu \mathrm{mol} / \mathrm{g})\end{array}$ & $\begin{array}{l}\mathrm{C} / \mathrm{N} \\
(\mathrm{mol} / \mathrm{mol})\end{array}$ & $\begin{array}{l}\mathbf{P}_{\mathrm{av}} \\
(\mu \mathrm{mol} / \mathrm{g})\end{array}$ & $\begin{array}{l}\text { NminS } \\
(\mathrm{kg} / \mathrm{ha} \\
\mathrm{yr})\end{array}$ & $\begin{array}{l}\text { NnitrS } \\
\text { (kg/ha } \\
\text { yr) }\end{array}$ \\
\hline Loja & BOM_LS1 & $\begin{array}{l}\text { Bombuscaro, } \\
\text { Podocarpus NP }\end{array}$ & 1020 & 1,81 & 3,60 & 1,50 & 1,34 & 0,86 & 82,50 & 15,08 & 1,08 & 99,25 & 11,07 \\
\hline Loja & BOM_LS2 & $\begin{array}{l}\text { Bombuscaro, } \\
\text { Podocarpus NP }\end{array}$ & 1026 & 3,38 & 3,60 & 1,50 & 1,34 & 0,86 & 82,50 & 15,08 & 0,95 & 96,90 & 126,45 \\
\hline Loja & BOM_LS3 & $\begin{array}{l}\text { Bombuscaro, } \\
\text { Podocarpus NP }\end{array}$ & 1046 & 0,50 & 4,67 & 2,17 & 8,61 & 62,63 & 2,22 & 12,08 & 0,63 & 95,33 & 43,53 \\
\hline Loja & BOM_LS4 & $\begin{array}{l}\text { Bombuscaro, } \\
\text { Podocarpus NP }\end{array}$ & 1054 & 2,31 & 4,88 & 10,22 & 67,97 & 282,24 & 1,93 & 13,47 & 1,73 & 8,51 & 75,69 \\
\hline Loja & BOM_LS5 & $\begin{array}{l}\text { Bombuscaro, } \\
\text { Podocarpus NP }\end{array}$ & 1056 & 1,63 & 4,88 & 10,22 & 67,97 & 282,24 & 1,93 & 13,47 & 2,21 & 179,52 & 230,58 \\
\hline Loja & BOM_LS6 & $\begin{array}{l}\text { Bombuscaro, } \\
\text { Podocarpus NP }\end{array}$ & 1044 & 5,38 & 4,32 & 3,29 & 40,58 & 232,14 & 2,30 & 14,93 & 2,87 & 318,04 & 362,09 \\
\hline Loja & BOM_MS1 & $\begin{array}{l}\text { Bombuscaro, } \\
\text { Podocarpus NP }\end{array}$ & 1132 & 0,25 & 3,72 & 1,89 & 1,64 & 7,06 & 52,52 & 13,23 & 0,92 & 244,37 & 156,17 \\
\hline Loja & BOM_MS2 & $\begin{array}{l}\text { Bombuscaro, } \\
\text { Podocarpus NP }\end{array}$ & 1069 & 2,94 & 3,22 & 0,99 & 1,42 & 1,93 & 97,58 & 13,26 & 1,10 & 153,92 & 133,84 \\
\hline Loja & BOM_MS3 & $\begin{array}{l}\text { Bombuscaro, } \\
\text { Podocarpus NP }\end{array}$ & 1049 & 1,22 & 3,83 & 2,27 & 7,31 & 25,15 & 41,67 & 13,61 & 1,23 & 41,85 & $-147,39$ \\
\hline Loja & BOM_MS4 & $\begin{array}{l}\text { Bombuscaro, } \\
\text { Podocarpus NP }\end{array}$ & 1140 & 7,19 & 4,84 & 4,20 & 40,49 & 250,30 & 0,51 & 10,91 & 2,96 & 44,53 & 46,75 \\
\hline Loja & BOM_MS5 & $\begin{array}{l}\text { Bombuscaro, } \\
\text { Podocarpus NP }\end{array}$ & 1170 & 1,63 & 5,59 & 7,20 & 42,43 & 203,99 & 0,23 & 15,06 & 1,85 & 45,19 & 48,23 \\
\hline Loja & BOM_MS6 & $\begin{array}{l}\text { Bombuscaro, } \\
\text { Podocarpus NP }\end{array}$ & 1129 & 2,19 & 5,12 & 1,59 & 59,12 & 119,16 & 0,24 & 11,39 & 2,09 & 140,59 & 128,68 \\
\hline Loja & BOM_US1 & $\begin{array}{l}\text { Bombuscaro, } \\
\text { Podocarpus NP }\end{array}$ & 1075 & 10,13 & 3,94 & 0,52 & 0,60 & 0,00 & 81,52 & 20,96 & 0,78 & 11,82 & 1,35 \\
\hline Loja & BOM_US2 & $\begin{array}{l}\text { Bombuscaro, } \\
\text { Podocarpus NP }\end{array}$ & 1066 & 12,06 & 3,65 & 1,04 & 0,80 & 0,70 & 144,05 & 32,56 & 1,17 & 12,77 & 8,20 \\
\hline Loja & BOM_US3 & $\begin{array}{l}\text { Bombuscaro, } \\
\text { Podocarpus NP }\end{array}$ & 1072 & 9,19 & 3,94 & 0,52 & 0,60 & 0,00 & 81,52 & 12,17 & 1,35 & 27,15 & 20,26 \\
\hline Loja & BOM_US4 & $\begin{array}{l}\text { Bombuscaro, } \\
\text { Podocarpus NP }\end{array}$ & 1268 & 2,56 & 3,18 & 1,42 & 1,86 & 0,96 & 162,47 & 17,90 & 1,74 & 125,67 & 109,96 \\
\hline
\end{tabular}




\begin{tabular}{|c|c|c|c|c|c|c|c|c|c|c|c|c|c|}
\hline Loja & BOM_US5 & $\begin{array}{l}\text { Bombuscaro, } \\
\text { Podocarpus NP }\end{array}$ & 1257 & 3,75 & 3,37 & 1,46 & 1,94 & 0,95 & 112,48 & 15,50 & 1,86 & 0,36 & $-34,12$ \\
\hline Loja & BOM_US6 & $\begin{array}{l}\text { Bombuscaro, } \\
\text { Podocarpus NP }\end{array}$ & 1266 & 1,13 & 3,37 & 1,46 & 1,94 & 0,95 & 112,48 & 15,50 & 1,80 & $-12,39$ & $-27,24$ \\
\hline Loja & CAJ_LS1 & $\begin{array}{l}\text { Cajanuma, } \\
\text { Podocarpus NP }\end{array}$ & 2818 & 7,50 & 2,80 & 1,28 & 1,00 & 0,68 & 16,78 & 12,04 & 4,01 & 12,69 & 0,00 \\
\hline Loja & CAJ_LS2 & $\begin{array}{l}\text { Cajanuma, } \\
\text { Podocarpus NP }\end{array}$ & 2805 & 6,75 & 2,80 & 1,28 & 1,00 & 0,68 & 16,78 & 12,04 & 2,55 & $-12,40$ & 0,00 \\
\hline Loja & CAJ_LS3 & $\begin{array}{l}\text { Cajanuma, } \\
\text { Podocarpus NP }\end{array}$ & 2798 & 8,19 & 3,50 & 2,23 & 2,75 & 3,50 & 73,43 & 12,29 & 1,86 & $-2,01$ & 0,00 \\
\hline Loja & CAJ_LS4 & $\begin{array}{l}\text { Cajanuma, } \\
\text { Podocarpus NP }\end{array}$ & 2865 & 5,00 & 2,81 & 3,53 & 5,27 & 2,26 & 66,93 & 12,44 & 2,84 & $-8,93$ & 0,00 \\
\hline Loja & CAJ_LS5 & $\begin{array}{l}\text { Cajanuma, } \\
\text { Podocarpus NP }\end{array}$ & 2878 & 4,08 & 2,81 & 3,53 & 5,27 & 2,26 & 66,93 & 12,44 & 2,84 & 9,27 & 0,00 \\
\hline Loja & CAJ_LS6 & $\begin{array}{l}\text { Cajanuma, } \\
\text { Podocarpus NP }\end{array}$ & 2880 & 7,88 & 2,92 & 2,19 & 2,14 & 0,20 & 9,09 & 11,57 & 3,21 & 41,32 & 0,00 \\
\hline Loja & CAJ_MS1 & $\begin{array}{l}\text { Cajanuma, } \\
\text { Podocarpus NP }\end{array}$ & 2859 & 6,94 & 2,96 & 2,02 & 2,76 & 0,88 & 102,78 & 13,25 & 1,15 & 15,78 & 0,00 \\
\hline Loja & CAJ_MS2 & $\begin{array}{l}\text { Cajanuma, } \\
\text { Podocarpus NP }\end{array}$ & 2872 & 13,31 & 2,78 & 4,15 & 2,39 & 1,45 & 66,00 & 18,62 & 1,41 & 7,54 & 0,00 \\
\hline Loja & CAJ_MS3 & $\begin{array}{l}\text { Cajanuma, } \\
\text { Podocarpus NP }\end{array}$ & 2844 & 17,13 & 2,96 & 2,02 & 2,76 & 0,88 & 102,78 & 13,25 & 1,80 & 16,76 & 8,95 \\
\hline Loja & CAJ_MS4 & $\begin{array}{l}\text { Cajanuma, } \\
\text { Podocarpus NP }\end{array}$ & 2900 & 10,63 & 3,05 & 1,83 & 1,48 & 0,66 & 41,34 & 14,03 & 2,55 & 17,69 & 0,00 \\
\hline Loja & CAJ_MS5 & $\begin{array}{l}\text { Cajanuma, } \\
\text { Podocarpus NP }\end{array}$ & 2885 & 15,19 & 2,77 & 3,22 & 2,27 & 0,55 & 16,44 & 16,25 & 0,74 & $-60,66$ & 0,00 \\
\hline Loja & CAJ_MS6 & $\begin{array}{l}\text { Cajanuma, } \\
\text { Podocarpus NP }\end{array}$ & 2875 & 18,31 & 2,77 & 3,22 & 2,27 & 0,55 & 16,44 & 17,26 & 1,58 & $-12,25$ & 0,00 \\
\hline Loja & CAJ_US1 & $\begin{array}{l}\text { Cajanuma, } \\
\text { Podocarpus NP }\end{array}$ & 2891 & 19,19 & 2,67 & 1,21 & 1,02 & 0,49 & 51,32 & 16,25 & 1,31 & $-25,51$ & 0,00 \\
\hline Loja & CAJ_US2 & $\begin{array}{l}\text { Cajanuma, } \\
\text { Podocarpus NP }\end{array}$ & 2885 & 12,63 & 2,89 & 1,85 & 1,93 & 1,22 & 12,77 & 19,57 & 0,98 & $-4,95$ & 0,00 \\
\hline Loja & CAJ_US3 & $\begin{array}{l}\text { Cajanuma, } \\
\text { Podocarpus NP }\end{array}$ & 2869 & 18,69 & 2,67 & 1,21 & 1,02 & 0,49 & 51,32 & 17,26 & 1,85 & 88,33 & 0,00 \\
\hline Loja & CAJ_US4 & $\begin{array}{l}\text { Cajanuma, } \\
\text { Podocarpus NP }\end{array}$ & 2886 & 22,81 & 2,62 & 3,31 & 2,48 & 0,94 & 20,77 & 21,40 & 0,64 & $-14,49$ & 0,00 \\
\hline Loja & CAJ_US5 & $\begin{array}{l}\text { Cajanuma, } \\
\text { Podocarpus NP }\end{array}$ & 2890 & 28,81 & 2,62 & 3,31 & 2,48 & 0,94 & 20,77 & 21,40 & 1,04 & 1,03 & 0,00 \\
\hline Loja & CAJ_US6 & $\begin{array}{l}\text { Cajanuma, } \\
\text { Podocarpus NP }\end{array}$ & 2893 & 34,75 & 3,00 & 0,93 & 0,50 & 0,23 & 117,68 & 18,87 & 1,15 & 22,03 & 0,00 \\
\hline Loja & SFM_LS1 & $\begin{array}{l}\text { San Francisco } \\
\text { Reserve }\end{array}$ & 2039 & 6,25 & 3,37 & 1,77 & 3,40 & 10,34 & 74,65 & 11,51 & 0,81 & $-1,04$ & 15,59 \\
\hline
\end{tabular}




\begin{tabular}{|c|c|c|c|c|c|c|c|c|c|c|c|c|c|}
\hline Loja & SFM_LS2 & $\begin{array}{l}\text { San Francisco } \\
\text { Reserve }\end{array}$ & 1993 & 5,09 & 3,73 & 2,06 & 11,09 & 27,61 & 20,52 & 11,82 & 1,67 & 54,59 & 60,69 \\
\hline Loja & SFM_LS3 & $\begin{array}{l}\text { San Francisco } \\
\text { Reserve }\end{array}$ & 2020 & 5,78 & 3,50 & 0,64 & 0,96 & 0,99 & 40,46 & 9,99 & 1,94 & 44,35 & $-1,87$ \\
\hline Loja & SFM_LS4 & $\begin{array}{l}\text { San Francisco } \\
\text { Reserve }\end{array}$ & 1913 & 4,44 & 3,52 & 1,00 & 3,95 & 4,48 & 21,34 & 8,30 & 1,33 & 83,03 & 91,52 \\
\hline Loja & SFM_LS5 & $\begin{array}{l}\text { San Francisco } \\
\text { Reserve }\end{array}$ & 1954 & 1,50 & 4,21 & 5,44 & 31,17 & 65,49 & 3,41 & 13,46 & 2,48 & 107,43 & $-19,05$ \\
\hline Loja & SFM_LS6 & $\begin{array}{l}\text { San Francisco } \\
\text { Reserve }\end{array}$ & 1933 & 0,72 & 4,21 & 5,44 & 31,17 & 65,49 & 3,41 & 15,47 & 2,50 & 86,38 & 37,03 \\
\hline Loja & SFM_MS1 & $\begin{array}{l}\text { San Francisco } \\
\text { Reserve }\end{array}$ & 2034 & 22,75 & 3,17 & 1,58 & 1,82 & 7,03 & 109,94 & 9,38 & 2,92 & 37,70 & 50,99 \\
\hline Loja & SFM_MS2 & $\begin{array}{l}\text { San Francisco } \\
\text { Reserve }\end{array}$ & 1950 & 7,81 & 5,14 & 7,56 & 42,53 & 60,14 & 0,28 & 15,85 & 4,45 & $-14,87$ & 59,10 \\
\hline Loja & SFM_MS3 & $\begin{array}{l}\text { San Francisco } \\
\text { Reserve }\end{array}$ & 2027 & 16,13 & 3,82 & 0,49 & 0,37 & 0,00 & 99,67 & 8,83 & 2,58 & 4,53 & 10,52 \\
\hline Loja & SFM_MS4 & $\begin{array}{l}\text { San Francisco } \\
\text { Reserve }\end{array}$ & 1927 & 2,75 & 4,46 & 2,42 & 29,59 & 49,80 & 11,14 & 10,23 & 3,32 & 163,12 & 157,82 \\
\hline Loja & SFM_MS5 & $\begin{array}{l}\text { San Francisco } \\
\text { Reserve }\end{array}$ & 1971 & 6,56 & 3,67 & 1,64 & 10,72 & 38,49 & 26,18 & 12,86 & 2,86 & 84,55 & 83,02 \\
\hline Loja & SFM_MS6 & $\begin{array}{l}\text { San Francisco } \\
\text { Reserve }\end{array}$ & 2020 & 16,94 & 3,12 & 2,17 & 3,75 & 4,27 & 79,79 & 13,46 & 1,87 & 27,40 & 36,86 \\
\hline Loja & SFM_US1 & $\begin{array}{l}\text { San Francisco } \\
\text { Reserve }\end{array}$ & 2002 & 19,92 & 2,91 & 0,61 & 0,87 & 0,62 & 26,26 & 15,45 & 1,96 & 78,00 & $-34,27$ \\
\hline Loja & SFM_US2 & $\begin{array}{l}\text { San Francisco } \\
\text { Reserve }\end{array}$ & 2026 & 35,94 & 3,02 & 0,42 & 0,14 & 1,63 & 31,32 & 13,26 & 1,10 & 48,50 & 0,00 \\
\hline Loja & SFM_US3 & $\begin{array}{l}\text { San Francisco } \\
\text { Reserve }\end{array}$ & 2089 & 22,79 & 3,00 & 0,42 & 0,38 & 0,54 & 72,96 & 11,97 & 0,64 & 70,57 & 0,00 \\
\hline Loja & SFM_US4 & $\begin{array}{l}\text { San Francisco } \\
\text { Reserve }\end{array}$ & 2063 & 17,69 & 2,72 & 0,89 & 0,64 & 0,25 & 85,97 & 14,69 & 0,69 & 83,12 & 38,20 \\
\hline Loja & SFM_US5 & $\begin{array}{l}\text { San Francisco } \\
\text { Reserve }\end{array}$ & 2054 & 21,94 & 2,61 & 1,37 & 0,98 & 0,46 & 64,98 & 16,12 & 0,48 & 36,84 & 0,00 \\
\hline Loja & SFM_US6 & $\begin{array}{l}\text { San Francisco } \\
\text { Reserve }\end{array}$ & 2039 & 31,13 & 2,61 & 1,37 & 0,98 & 0,46 & 64,98 & 16,12 & 0,71 & 12,13 & 0,00 \\
\hline Napo & YAY_01 & $\begin{array}{l}\text { Yanayacu } \\
\text { biological station, } \\
\text { Reserva Biósfera } \\
\text { Sumaco }\end{array}$ & 2085 & 7,13 & 4,73 & 1,76 & 12,54 & 151,67 & 46,43 & 11,34 & 4,86 & 171,55 & 38,69 \\
\hline Napo & YAY_02 & $\begin{array}{l}\text { Yanayacu } \\
\text { biological station, } \\
\text { Reserva Biósfera } \\
\text { Sumaco }\end{array}$ & 2080 & 3,38 & 4,44 & 2,34 & 3,22 & 15,78 & 84,55 & 12,35 & 2,96 & 482,17 & 427,42 \\
\hline
\end{tabular}




\begin{tabular}{|c|c|c|c|c|c|c|c|c|c|c|c|c|c|}
\hline Napo & YAY_03 & $\begin{array}{l}\text { Yanayacu } \\
\text { biological station, } \\
\text { Reserva Biósfera } \\
\text { Sumaco }\end{array}$ & 2055 & 6,00 & 4,30 & 0,83 & 3,02 & 24,57 & 82,89 & 12,14 & 1,06 & 567,21 & 558,45 \\
\hline Napo & YAY_04 & $\begin{array}{l}\text { Yanayacu } \\
\text { biological station, } \\
\text { Reserva Biósfera } \\
\text { Sumaco }\end{array}$ & 2070 & 6,00 & 4,33 & 2,15 & 2,53 & 19,71 & 62,04 & 12,24 & 0,81 & 306,60 & 341,27 \\
\hline Napo & YAY_05 & $\begin{array}{l}\text { Yanayacu } \\
\text { biological station, } \\
\text { Reserva Biósfera } \\
\text { Sumaco }\end{array}$ & 2080 & 3,81 & 4,24 & 2,69 & 5,49 & 52,56 & 85,48 & 11,47 & 3,18 & 403,69 & 407,70 \\
\hline Napo & GUA_06 & $\begin{array}{l}\text { Cordillera } \\
\text { Guacamayos, } \\
\text { Reserva Ecológica } \\
\text { Antisana } \\
\end{array}$ & 2000 & 5,00 & 4,55 & 1,50 & 2,44 & 17,80 & 24,50 & 11,13 & 3,30 & 205,13 & 178,49 \\
\hline Napo & GUA_07 & $\begin{array}{l}\text { Cordillera } \\
\text { Guacamayos, } \\
\text { Reserva Ecológica } \\
\text { Antisana }\end{array}$ & 1940 & 4,00 & 4,38 & 2,57 & 2,24 & 12,02 & 61,00 & 11,94 & 0,49 & 73,36 & 141,99 \\
\hline Napo & GUA_08 & $\begin{array}{l}\text { Cordillera } \\
\text { Guacamayos, } \\
\text { Reserva Ecológica } \\
\text { Antisana } \\
\end{array}$ & 1995 & 2,94 & 4,40 & 1,56 & 1,83 & 14,34 & 10,35 & 11,59 & 1,05 & 244,91 & 231,41 \\
\hline Napo & SUM_09 & $\begin{array}{l}\text { Sumaco volcano, } \\
\text { PN Sumaco- } \\
\text { Galeras }\end{array}$ & 1920 & 4,94 & 4,54 & 2,33 & 10,34 & 76,22 & 21,93 & 10,76 & 1,17 & 465,74 & 379,60 \\
\hline Napo & SUM_10 & $\begin{array}{l}\text { Sumaco volcano, } \\
\text { PN Sumaco- } \\
\text { Galeras }\end{array}$ & 1940 & 6,50 & 4,57 & 0,82 & 1,54 & 11,32 & 46,69 & 11,52 & 0,83 & 500,05 & 483,63 \\
\hline Napo & SUM_11 & $\begin{array}{l}\text { Sumaco volcano, } \\
\text { PN Sumaco- } \\
\text { Galeras }\end{array}$ & 1950 & 8,25 & 4,70 & 0,11 & 0,84 & 6,30 & 10,63 & 10,50 & 0,54 & 446,76 & 446,76 \\
\hline Napo & SUM_12 & $\begin{array}{l}\text { Sumaco volcano, } \\
\text { PN Sumaco- } \\
\text { Galeras }\end{array}$ & 2015 & 3,75 & 4,36 & 3,94 & 9,04 & 52,33 & 13,51 & 11,03 & 0,16 & 645,68 & 523,04 \\
\hline Napo & SUM_13 & $\begin{array}{l}\text { Sumaco volcano, } \\
\text { PN Sumaco- } \\
\text { Galeras }\end{array}$ & 2000 & 5,19 & 4,44 & 2,96 & 3,06 & 22,40 & 4,44 & 11,50 & 2,19 & 251,12 & 106,58 \\
\hline Napo & SUM_14 & $\begin{array}{l}\text { Sumaco volcano, } \\
\text { PN Sumaco- } \\
\text { Galeras }\end{array}$ & 1580 & 4,69 & 4,34 & 2,81 & 6,95 & 89,86 & 33,71 & 11,74 & 2,26 & 325,94 & 347,12 \\
\hline
\end{tabular}




\begin{tabular}{|c|c|c|c|c|c|c|c|c|c|c|c|c|c|}
\hline Napo & SUM_15 & $\begin{array}{l}\text { Sumaco volcano, } \\
\text { PN Sumaco- } \\
\text { Galeras }\end{array}$ & 1590 & 5,00 & 4,21 & 1,95 & 4,12 & 23,97 & 53,01 & 12,39 & 2,20 & 372,30 & 464,28 \\
\hline Napo & SUM_16 & $\begin{array}{l}\text { Sumaco volcano, } \\
\text { PN Sumaco- } \\
\text { Galeras }\end{array}$ & 1610 & 4,13 & 4,35 & 1,49 & 7,26 & 29,24 & 35,25 & 11,65 & 0,18 & 588,01 & 487,64 \\
\hline Napo & SUM_17 & $\begin{array}{l}\text { Sumaco volcano, } \\
\text { PN Sumaco- } \\
\text { Galeras }\end{array}$ & 1610 & 4,25 & 4,42 & 1,81 & 9,66 & 40,44 & 44,35 & 11,48 & 0,96 & 297,48 & 335,80 \\
\hline Napo & SUM_18 & $\begin{array}{l}\text { Sumaco volcano, } \\
\text { PN Sumaco- } \\
\text { Galeras }\end{array}$ & 1590 & 5,63 & 4,42 & 1,64 & 5,66 & 20,23 & 31,92 & 11,76 & 0,29 & 314,63 & 357,70 \\
\hline Napo & SUM_19 & $\begin{array}{l}\text { Sumaco volcano, } \\
\text { PN Sumaco- } \\
\text { Galeras }\end{array}$ & 1590 & 5,88 & 4,41 & 1,79 & 5,43 & 22,55 & 29,20 & 11,42 & 0,15 & 577,79 & 505,89 \\
\hline Napo & GAL_20 & $\begin{array}{l}\text { Cordillera } \\
\text { Galeras, PN } \\
\text { Sumaco-Galeras }\end{array}$ & 1090 & 2,50 & 4,20 & 2,24 & 10,06 & 71,95 & 21,42 & 10,87 & 1,21 & 497,86 & 526,70 \\
\hline Napo & GAL_21 & $\begin{array}{l}\text { Cordillera } \\
\text { Galeras, PN } \\
\text { Sumaco-Galeras }\end{array}$ & 1080 & 2,38 & 4,87 & 2,66 & 14,36 & 237,64 & 8,99 & 9,90 & 0,76 & 119,72 & 73,73 \\
\hline Napo & GAL_22 & $\begin{array}{l}\text { Cordillera } \\
\text { Galeras, PN } \\
\text { Sumaco-Galeras }\end{array}$ & 1060 & 2,19 & 4,02 & 1,90 & 7,27 & 45,94 & 53,52 & 11,16 & 0,24 & 23,36 & 319,01 \\
\hline Napo & GAL_23 & $\begin{array}{l}\text { Cordillera } \\
\text { Galeras, PN } \\
\text { Sumaco-Galeras }\end{array}$ & 1090 & 2,25 & 3,97 & 1,81 & 4,37 & 14,85 & 54,96 & 12,02 & 0,32 & 243,09 & 283,61 \\
\hline Napo & GAL_24 & $\begin{array}{l}\text { Cordillera } \\
\text { Galeras, PN } \\
\text { Sumaco-Galeras }\end{array}$ & 1110 & 1,44 & 4,32 & 3,09 & 4,86 & 16,46 & 60,64 & 13,36 & 0,89 & 448,95 & 299,67 \\
\hline Napo & GAL_25 & $\begin{array}{l}\text { Cordillera } \\
\text { Galeras, PN } \\
\text { Sumaco-Galeras }\end{array}$ & 1450 & 8,25 & 4,57 & 1,35 & 1,51 & 6,00 & 20,39 & 13,68 & 0,25 & $-307,33$ & 32,85 \\
\hline Napo & GAL_26 & $\begin{array}{l}\text { Cordillera } \\
\text { Galeras, PN } \\
\text { Sumaco-Galeras }\end{array}$ & 1560 & 5,31 & 4,04 & 2,39 & 2,00 & 8,50 & 75,91 & 14,86 & 0,26 & 422,31 & 454,43 \\
\hline Napo & GUA_27 & $\begin{array}{l}\text { Cordillera } \\
\text { Guacamayos, } \\
\text { Reserva Ecológica } \\
\text { Antisana }\end{array}$ & 1990 & 6,88 & 4,52 & 1,89 & 2,07 & 12,74 & 49,50 & 12,87 & 0,09 & 300,03 & 123,73 \\
\hline
\end{tabular}




\begin{tabular}{|c|c|c|c|c|c|c|c|c|c|c|c|c|c|}
\hline Napo & GAL_28 & $\begin{array}{l}\text { Cordillera } \\
\text { Galeras, PN } \\
\text { Sumaco-Galeras }\end{array}$ & 1597 & 5,63 & 3,78 & 2,71 & 5,19 & 17,66 & 46,73 & 16,01 & 0,04 & 228,49 & 132,50 \\
\hline Napo & GAL_29 & $\begin{array}{l}\text { Cordillera } \\
\text { Galeras, PN } \\
\text { Sumaco-Galeras }\end{array}$ & 1050 & 1,13 & 4,14 & 2,18 & 2,07 & 11,60 & 84,76 & 11,69 & 0,98 & 507,71 & 601,15 \\
\hline Napo & HAM_42 & $\begin{array}{l}\text { Hakuna Matata, } \\
\text { Reserva Biósfera } \\
\text { Sumaco }\end{array}$ & 970 & 1,31 & 4,02 & 4,05 & 7,91 & 20,84 & 44,67 & 11,66 & 0,38 & 31,02 & 33,58 \\
\hline Napo & HAM_43 & $\begin{array}{l}\text { Hakuna Matata, } \\
\text { Reserva Biósfera } \\
\text { Sumaco }\end{array}$ & 960 & 1,56 & 4,12 & 3,35 & 10,18 & 14,31 & 50,53 & 10,98 & 0,37 & 26,28 & 23,73 \\
\hline Napo & HAM_44 & $\begin{array}{l}\text { Hakuna Matata, } \\
\text { Reserva Biósfera } \\
\text { Sumaco }\end{array}$ & 1000 & 1,75 & 4,18 & 3,31 & 18,47 & 23,74 & 39,68 & 11,09 & 0,30 & 5,84 & 13,14 \\
\hline Napo & HAM_45 & $\begin{array}{l}\text { Hakuna Matata, } \\
\text { Reserva Biósfera } \\
\text { Sumaco }\end{array}$ & 1050 & 2,25 & 3,99 & 2,22 & 8,28 & 13,35 & 63,17 & 11,27 & 0,48 & 25,55 & 44,53 \\
\hline Napo & HAM_46 & $\begin{array}{l}\text { Hakuna Matata, } \\
\text { Reserva Biósfera } \\
\text { Sumaco } \\
\end{array}$ & 1020 & 1,94 & 4,02 & 3,17 & 6,28 & 17,27 & 38,56 & 10,97 & 0,05 & $-15,33$ & 1,82 \\
\hline Napo & GAL_47 & $\begin{array}{l}\text { Cordillera } \\
\text { Galeras, PN } \\
\text { Sumaco-Galeras }\end{array}$ & 1000 & 0,75 & 4,39 & 2,32 & 9,23 & 104,67 & 15,47 & 12,73 & 0,13 & $-67,53$ & 86,50 \\
\hline Napo & GAL_48 & $\begin{array}{l}\text { Cordillera } \\
\text { Galeras, PN } \\
\text { Sumaco-Galeras }\end{array}$ & 1130 & 6,13 & 4,43 & 2,48 & 5,51 & 24,38 & 70,66 & 11,30 & 0,27 & 428,14 & 515,75 \\
\hline Napo & GAL_49 & $\begin{array}{l}\text { Cordillera } \\
\text { Galeras, PN } \\
\text { Sumaco-Galeras }\end{array}$ & 1080 & 1,63 & 4,33 & 2,85 & 9,78 & 91,84 & 37,09 & 13,75 & 0,15 & 1076,02 & 1055,58 \\
\hline Napo & GAL_50 & $\begin{array}{l}\text { Cordillera } \\
\text { Galeras, PN } \\
\text { Sumaco-Galeras }\end{array}$ & 1557 & 4,63 & 3,85 & 1,61 & 5,04 & 16,31 & 45,04 & 13,32 & 0,14 & 492,38 & 180,68 \\
\hline Napo & HAM_51 & $\begin{array}{l}\text { Hakuna Matata, } \\
\text { Reserva Biósfera } \\
\text { Sumaco }\end{array}$ & 1080 & 0,69 & 4,32 & 1,84 & 6,62 & 18,00 & 62,95 & 12,78 & 0,20 & 35,77 & 37,96 \\
\hline Napo & GUA_52 & $\begin{array}{l}\text { Cordillera } \\
\text { Guacamayos, } \\
\text { Reserva Ecológica } \\
\text { Antisana }\end{array}$ & 1980 & 5,50 & 4,53 & 2,05 & 6,22 & 22,25 & 18,95 & 12,35 & 0,26 & 59,13 & 65,70 \\
\hline Napo & GUA_53 & $\begin{array}{l}\text { Cordillera } \\
\text { Guacamayos, }\end{array}$ & 1980 & 5,31 & 4,59 & 1,18 & 5,75 & 20,85 & 20,35 & 12,28 & 0,25 & 56,21 & 112,42 \\
\hline
\end{tabular}




\begin{tabular}{|c|c|c|c|c|c|c|c|c|c|c|c|c|c|}
\hline & & $\begin{array}{l}\text { Reserva Ecológica } \\
\text { Antisana }\end{array}$ & & & & & & & & & & & \\
\hline Napo & GUA_54 & $\begin{array}{l}\text { Cordillera } \\
\text { Guacamayos, } \\
\text { Reserva Ecológica } \\
\text { Antisana }\end{array}$ & 2000 & 4,25 & 4,41 & 1,61 & 5,73 & 16,84 & 51,70 & 12,01 & 0,07 & 273,75 & 270,10 \\
\hline Napo & GUA_55 & $\begin{array}{l}\text { Cordillera } \\
\text { Guacamayos, } \\
\text { Reserva Ecológica } \\
\text { Antisana } \\
\end{array}$ & 2000 & 6,25 & 4,41 & 1,06 & 4,93 & 10,94 & 46,93 & 12,98 & 0,21 & 125,19 & 128,84 \\
\hline Napo & SUM_56 & $\begin{array}{l}\text { Sumaco volcano, } \\
\text { PN Sumaco- } \\
\text { Galeras }\end{array}$ & 1931 & 6,88 & 4,55 & 1,31 & 5,18 & 18,21 & 33,24 & 11,05 & 0,42 & 375,22 & 288,35 \\
\hline Napo & SUM_57 & $\begin{array}{l}\text { Sumaco volcano, } \\
\text { PN Sumaco- } \\
\text { Galeras }\end{array}$ & 2000 & 5,00 & 4,41 & 2,45 & 6,61 & 28,14 & 34,52 & 14,02 & 0,05 & 266,81 & 347,12 \\
\hline Napo & SUM_58 & $\begin{array}{l}\text { Sumaco volcano, } \\
\text { PN Sumaco- } \\
\text { Galeras }\end{array}$ & 1580 & 7,56 & 4,52 & 0,86 & 3,96 & 17,71 & 46,55 & 12,29 & 0,39 & 127,75 & 236,16 \\
\hline Napo & SUM_59 & $\begin{array}{l}\text { Sumaco volcano, } \\
\text { PN Sumaco- } \\
\text { Galeras }\end{array}$ & 1600 & 5,13 & 4,22 & 2,93 & 4,73 & 21,91 & 34,49 & 12,18 & 0,33 & $-322,66$ & 87,23 \\
\hline Napo & GAL_60 & $\begin{array}{l}\text { Cordillera } \\
\text { Galeras, PN } \\
\text { Sumaco-Galeras }\end{array}$ & 1570 & 11,00 & 4,23 & 1,55 & 5,05 & 21,80 & 59,74 & 15,24 & 0,13 & 520,49 & 435,08 \\
\hline Napo & GAL_61 & $\begin{array}{l}\text { Cordillera } \\
\text { Galeras, PN } \\
\text { Sumaco-Galeras }\end{array}$ & 1560 & 9,38 & 4,45 & 1,57 & 5,87 & 24,44 & 65,13 & 15,08 & 0,10 & 118,99 & 147,82 \\
\hline Napo & GAL_62 & $\begin{array}{l}\text { Cordillera } \\
\text { Galeras, PN } \\
\text { Sumaco-Galeras }\end{array}$ & 1590 & 11,00 & 4,38 & 1,63 & 5,54 & 23,81 & 70,30 & 15,31 & 0,04 & 209,51 & 127,39 \\
\hline Napo & RHO_63 & $\begin{array}{l}\text { Rio Hollín, } \\
\text { Reserva Biósfera } \\
\text { Sumaco }\end{array}$ & 1190 & 4,38 & 4,36 & 1,54 & 4,52 & 15,48 & 60,88 & 12,67 & 0,13 & 539,84 & 501,88 \\
\hline Napo & RHO_64 & $\begin{array}{l}\text { Rio Hollín, } \\
\text { Reserva Biósfera } \\
\text { Sumaco }\end{array}$ & 1195 & 4,56 & 4,43 & 1,33 & 4,98 & 16,70 & 59,17 & 13,10 & 0,00 & 320,11 & 402,60 \\
\hline Napo & RHO_65 & $\begin{array}{l}\text { Rio Hollín, } \\
\text { Reserva Biósfera } \\
\text { Sumaco }\end{array}$ & 1210 & 3,38 & 4,36 & 2,24 & 4,19 & 14,13 & 64,12 & 13,21 & 0,14 & 485,45 & 472,68 \\
\hline
\end{tabular}




\begin{tabular}{|c|c|c|c|c|c|c|c|c|c|c|c|c|c|}
\hline Napo & RHO_66 & $\begin{array}{l}\text { Rio Hollín, } \\
\text { Reserva Biósfera } \\
\text { Sumaco }\end{array}$ & 1165 & 3,63 & 4,41 & 2,72 & 5,15 & 18,97 & 179,19 & 12,42 & 0,06 & 521,95 & 520,13 \\
\hline Napo & RHO_67 & $\begin{array}{l}\text { Rio Hollín, } \\
\text { Reserva Biósfera } \\
\text { Sumaco }\end{array}$ & 1180 & 2,19 & 4,27 & 3,55 & 7,06 & 33,57 & 73,65 & 11,39 & 0,05 & 964,33 & 903,38 \\
\hline Napo & COC_76 & $\begin{array}{l}\text { Cocodrilos, } \\
\text { Reserva Biósfera } \\
\text { Sumaco }\end{array}$ & 1550 & 6,13 & 4,57 & 0,94 & 1,07 & 9,75 & 48,41 & 12,46 & 0,12 & 334,34 & 417,56 \\
\hline Napo & COC_77 & $\begin{array}{l}\text { Cocodrilos, } \\
\text { Reserva Biósfera } \\
\text { Sumaco }\end{array}$ & 1490 & 5,25 & 4,60 & 1,57 & 7,66 & 25,13 & 59,76 & 13,56 & 0,02 & 241,63 & 285,06 \\
\hline Napo & COC_78 & $\begin{array}{l}\text { Cocodrilos, } \\
\text { Reserva Biósfera } \\
\text { Sumaco }\end{array}$ & 1490 & 5,06 & 4,54 & 0,77 & 1,26 & 6,59 & 38,52 & 13,16 & 0,15 & 220,09 & 260,25 \\
\hline Napo & COC_79 & $\begin{array}{l}\text { Cocodrilos, } \\
\text { Reserva Biósfera } \\
\text { Sumaco }\end{array}$ & 1490 & 7,81 & 4,66 & 0,60 & 1,03 & 7,26 & 21,32 & 12,81 & 0,03 & 274,85 & 292,00 \\
\hline Napo & COC_80 & $\begin{array}{l}\text { Cocodrilos, } \\
\text { Reserva Biósfera } \\
\text { Sumaco }\end{array}$ & 1570 & 4,69 & 4,54 & 0,83 & 1,22 & 6,01 & 26,82 & 11,78 & 0,56 & 302,58 & 392,38 \\
\hline Napo & YAY_87 & $\begin{array}{l}\text { Yanayacu } \\
\text { biological station, } \\
\text { Reserva Biósfera } \\
\text { Sumaco }\end{array}$ & 2420 & 8,13 & 4,25 & 0,97 & 2,13 & 8,72 & 74,83 & 15,37 & 0,13 & NA & NA \\
\hline Napo & YAY_88 & $\begin{array}{l}\text { Yanayacu } \\
\text { biological station, } \\
\text { Reserva Biósfera } \\
\text { Sumaco } \\
\end{array}$ & 2409 & 6,13 & 4,35 & 0,51 & 2,94 & 21,81 & 38,18 & 11,95 & 0,27 & NA & NA \\
\hline Napo & YAY_89 & $\begin{array}{l}\text { Yanayacu } \\
\text { biological station, } \\
\text { Reserva Biósfera } \\
\text { Sumaco }\end{array}$ & 2409 & 5,88 & 4,43 & 0,59 & 1,94 & 18,19 & 33,09 & 13,45 & 0,30 & NA & NA \\
\hline Napo & GUA_90 & Guango Reserve & 2890 & 4,50 & 4,20 & 1,22 & 4,07 & 36,73 & 60,53 & 12,75 & 0,33 & NA & NA \\
\hline Napo & GUA_91 & Guango Reserve & 3098 & 11,63 & 4,94 & 2,56 & 56,92 & 406,02 & 17,82 & 13,54 & 0,42 & NA & NA \\
\hline Napo & GUA_92 & Guango Reserve & 3098 & 8,25 & 4,64 & 4,92 & 49,53 & 374,90 & 15,53 & 14,61 & 0,77 & NA & NA \\
\hline
\end{tabular}


